\title{
Obstrução nasolacrimal congênita: fatores relacionados com a possibilidade de cura
}

\author{
Congenital nasolacrimal obstruction:successful treatment factors
}

\author{
Silvana Artioli Schellini ${ }^{1}$ \\ Magda Massae Hata Viveiros ${ }^{2}$ \\ Elisângela Jaqueta ${ }^{3}$ \\ Carlos Roberto Padovani ${ }^{4}$ \\ Carlos Roberto Pereira Padovani ${ }^{5}$
}

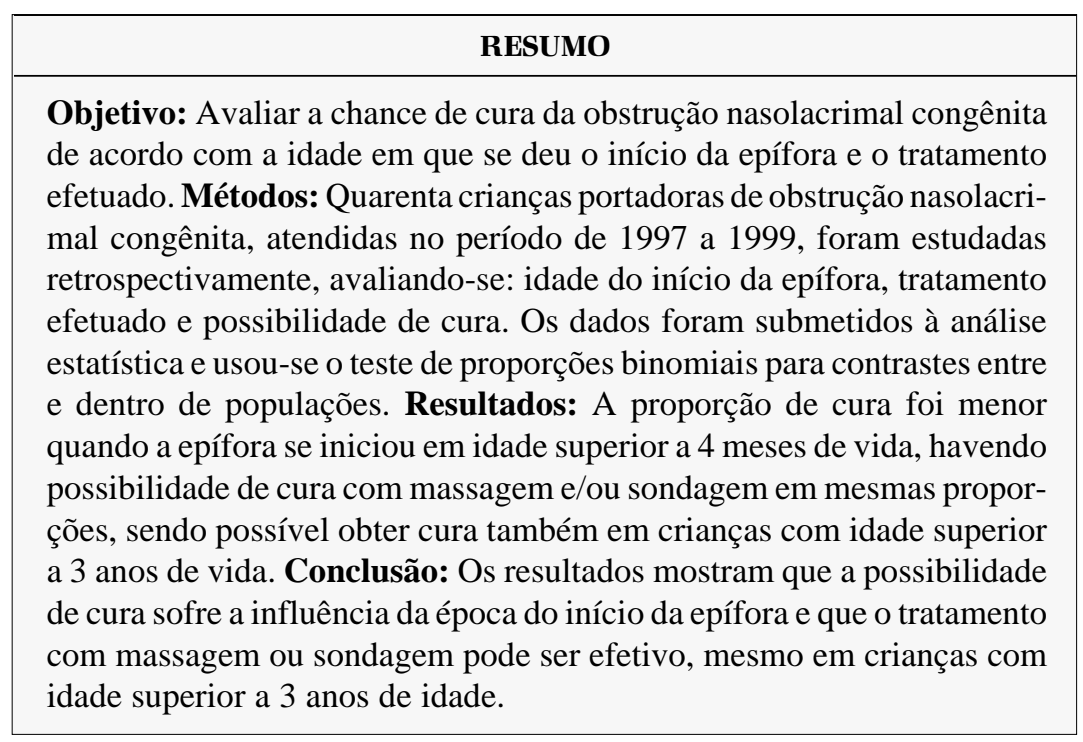

Descritores: Obstrução dos ductos lacrimais/congênito; Obstrução dos ductos lacrimais/ terapia; Ducto nasolacrimal; Massagem; Resultado de tratamento
Trabalho realizado na Faculdade de Medicina de Botucatu da Universidade Estadual Paulista - UNESP.

${ }^{1}$ Professora Livre-Docente do Departamento de Oftalmologia, Otorrinolaringologia e Cirurgia de Cabeça e Pescoço da Faculdade de Medicina de Botucatu da Universidade Estadual Paulista - UNESP. Botucatu (SP).

${ }^{2}$ Pós-graduanda da Faculdade de Medicina de Botucatu da UNESP. Botucatu (SP).

${ }^{3}$ Ex-residente de Oftalmologia do Departamento de Oftalmologia, Otorrinolaringologia e Cirurgia de Cabeça e Pescoço da Faculdade de Medicina de Botucatu da UNESP. Botucatu (SP).

${ }^{4}$ Professor Titular do Departamento de Bioestatística do Instituto de Biociências da UNESP. Botucatu (SP).

Pós-graduando da Faculdade de Ciências Agronômicas da UNESP. Botucatu (SP).

Endereço para correspondência: Silvana Artioli Schellini - Dep. OFT/ORL/CCP - Faculdade de Medicina de Botucatu - UNESP - Botucatu (SP) CEP 18618-970 E-mail: sartioli@fmb.unesp.br

Recebido para publicação em 07.05.2004

Versão revisada recebida em 29.09.2004

Aprovação em 22.10.2004

Nota Editorial: Após concluída a análise do artigo sob sigilo editorial e com a anuência dos Drs. Ana Estela B. P. Ponce Sant'Anna e Marcos Carvalho da Cunha sobre a divulgação de seus nomes como revisores dele, agradecemos suas participações nesse processo.

\section{INTRODUÇ̃̃̃O}

Dentre as alterações congênitas que ocorrem na via lacrimal excretora, a obstrução nasolacrimal congênita (ONLC) é a mais comum, responsável por aproximadamente $90 \%$ das obstruções existentes, podendo acometer 6 a $20 \%$ dos recém-nascidos ${ }^{(1-2)}$. Em crianças brasileiras, a incidência de ONLC é de $2,7 \%{ }^{(3)}$.

As complicações são raras e a resolução espontânea até um ano de idade é a regra em aproximadamente $95 \%$ das crianças ${ }^{(4-5)}$.

Existem controvérsias quanto a melhor época para a realização da sondagem lacrimal, havendo duas correntes, uma que defende a sondagem precoce, realizada em recém-natos, no próprio consultório, assim que é detectado o problema, por assim não haver necessidade de anestesia geral ${ }^{(6-7)}$.

Os defensores da sondagem tardia, baseados nos altos índices de cura espontânea, recomendam que se aguarde até a idade de 1 ano, realizando massagem na região do saco e ducto nasolacrimal, evitando, assim, procedimentos desnecessários e diminuindo o risco de trauma canalicular, com formação de falsa via ${ }^{(8-9)}$.

Nos últimos anos, estudos mostram a possibilidade de cura também em crianças mais velhas ${ }^{(8,10)}$, acrescentando mais controvérsia sobre a época ideal para o tratamento da ONLC.

Apesar da possibilidade de cura da ONLC estar fortemente associada 
com a idade em que a sondagem é realizada, existem outros fatores que podem influenciar os índices de cura, tais como o local em que ocorre a obstrução e o grau de dilatação do saco lacrimal ${ }^{(11)}$.

O presente estudo teve por objetivo identificar outros fatores que pudessem influenciar os índices de cura da ONLC em crianças.

\section{MÉTODOS}

Foi feito estudo retrospectivo do qual participaram 40 crianças portadoras de ONLC, atendidas consecutivamente no Ambulatório de Vias Lacrimais da Faculdade de Medicina de Botucatu - UNESP, no período de 1997 a 1999. Foram avaliadas: idade de início da epífora, idade na primeira consulta, tratamento efetuado conforme a conduta do serviço que é a realização de massagem de Criegler até 1 ano de vida, passando-se a sondagem. A sondagem precoce está reservada para os casos de mucoceles e crianças que já tiveram dacriocistite aguda. As crianças que não se curam com a sondagem são operadas (entubação ou dacriocistorrinostomia).

Usando o teste de proporções binomiais para avaliação de contraste entre e dentro de populações, procurou-se determinar a possibilidade de cura com os tratamentos empregados, de acordo com a idade do início da epífora. As conclusões foram tiradas no nível de $5 \%$ de significância, apontadas nas tabelas por meio de letras, de modo que duas letras iguais indicam não haver diferença estatística; as letras minúsculas comparam variáveis nas colunas e as maiúsculas, nas linhas.

\section{RESULTADOS}

Das 40 crianças que compuseram a amostra do estudo, em $57,5 \%$ a queixa de epífora surgiu com menos de um mês de idade, sendo a distribuição não casual (Tabela 1). 50,0\% delas procuraram tratamento com menos de 6 meses de idade, com distribuição casual (Tabela 2).

O tratamento instituído foi baseado na massagem de Criegler (45,0\%), mantida até 1 ano de vida. A partir desta época, quando a epífora se manteve, foi realizada a sondagem terapêutica, o que ocorreu em $52,5 \%$ das crianças. Houve necessidade de cirurgia em apenas uma criança $(2,0 \%)$ (Tabela 3$)$.

Com relação aos índices de cura, observou-se que existe maior chance de resolução da epífora em crianças maiores do que 6 meses, com distribuição casual a partir desta idade, até por volta dos 3 anos $\left(X^{2}=8,8\right)$ (Tabela 4$)$.

Avaliando-se a proporção de cura com a massagem em relação à idade do início da epífora, os maiores índices de resolução ocorreram em crianças que apresentavam a queixa iniciando-se antes dos 2 meses de vida. A probabilidade de não-cura foi maior nas crianças cuja epífora se iniciou após os 4 meses de vida (Tabela 5).

Com a sondagem, a proporção de cura foi semelhante em todas as faixas etárias avaliadas; porém, houve predomínio de insucesso quando o início da epífora se deu após os 4 meses de vida (Tabela 6).

A tabela 7 apresenta o teste de proporções binomiais para a avaliação da resposta ao tratamento - massagem ou sondagem - com relação ao resultado obtido, observou-se que a chance de cura com a massagem foi semelhante à resolução com a sondagem.

O gráfico 1 apresenta a porcentagem de cura de acordo com a idade da criança e idade de início da epífora, mostrando que altos índices de cura ocorrem mesmo em crianças acima dos 3 anos de idade.

\section{DISCUSS $\tilde{O} O$}

A produção de lágrimas inicia-se na primeira ou na segunda semana de vida, o que condiciona o surgimento da epífora em portadores de ONLC a esta época. Assim, a maioria das crianças desta amostra apresentavam o início da epífora em idade inferior a 1 mês. Algumas, porém, possuíam o sintoma relatado mais tardiamente. A razão que poderia explicar isso seria ser o fato da epífora ser um sintoma que não atrai muito a

\begin{tabular}{|lcc|}
\hline \multicolumn{3}{|c|}{$\begin{array}{c}\text { Tabela 1. Distribuição de portadores de ONLC segundo época do } \\
\text { aparecimento da epífora }\end{array}$} \\
Idade do inicio da epífora & No de pacientes & $\%$ \\
$<1$ mês & 23 & 57,5 \\
2 meses & 5 & 12,5 \\
3 meses & 6 & 15,0 \\
$\geq 4$ meses & 6 & 15,0 \\
Total & 40 & 100,0 \\
$X^{2}=36(p>0,001)$ & & \\
\hline
\end{tabular}

$\begin{aligned} & \text { Tabela 2. Distribuição dos portadores de ONLC segundo idade da } \\
& \text { consulta }\end{aligned}$
\begin{tabular}{lcc} 
Idade da consulta & $N o$ de pacientes & $\%$ \\
$\leq 6$ meses & 20 & 50,0 \\
6 meses - 1 ano & 10 & 25,0 \\
$\geq 1$ ano & 10 & 25,0 \\
Total & 40 & 100,0 \\
$X^{2}=5,01$ ( $\left.p>0,05\right)$ : distribuição casual & & \\
\hline
\end{tabular}

\begin{tabular}{|lcr|}
\hline \multicolumn{3}{|c|}{ Tabela 3. Distribuição dos portadores de ONLC de acordo com o } \\
tratamento efetuado \\
Tratamento & No pacientes & $\%$ \\
Massagem & 18 & 45,0 \\
Sondagem & 21 & 52,5 \\
Cirurgia & 1 & 2,0 \\
Total & 40 & 100,0 \\
$X^{2}=17,5(p<0,001) ;($ sondagem = massagem) $>$ cirurgia & \\
\hline
\end{tabular}




\begin{tabular}{|lcr|}
\hline \multicolumn{3}{|c|}{ Tabela 4. Distribuição dos portadores de ONLC segundo idade } \\
da cura \\
Idade da cura & No pacientes & $\%$ \\
$<6$ meses & 1 & 2,5 \\
6 meses - 1 ano & 8 & 20,0 \\
1 ano - 2 anos & 12 & 30,0 \\
2 anos - 3 anos & 9 & 22,5 \\
$\geq 3$ anos & 10 & 25,0 \\
Total & 40 & 100,0 \\
\hline
\end{tabular}

Tabela 5. Proporção de cura com massagem segundo idade do início da epífora

\begin{tabular}{lcccr} 
& \multicolumn{2}{c}{ Cura } & \\
\cline { 2 - 4 } Início & Sim & Não & Total \\
$<1$ mês & 0,478 ab A & 0,522 ab A & 23 \\
2 meses & 0,800 b B & 0,200 a A & 5 \\
3 meses & 0,333 ab A & 0,667 ab A & 6 \\
$\geq 4$ meses & 0,167 a A & 0,833 b B & 6
\end{tabular}

Letras minúsculas comparam as variáveis que estão nas colunas e as maiúsculas, as das linhas. Letras iguais apontam não significância e as diferentes, que houve significância estatística entre os dados obtidos

\begin{tabular}{|l}
$\begin{array}{l}\text { Tabela 6. Proporção de cura com sondagem segundo idade do } \\
\text { início da epífora }\end{array}$ \\
$\begin{array}{lccc}\text { Início } & \text { Cura } & \text { Total } \\
<1 \text { mês } & 0,522 \text { b A } & 0,478 \text { a A } & 23 \\
2 \text { meses } & 0,500 \text { a A } & 0,500 \text { a A } & 5 \\
3 \text { meses } & 0,667 \text { b A } & 0,333 \text { a A } & 6 \\
\geq 4 \text { meses } & 0,833 \text { b B } & 0,167 \text { a A } & 6 \\
\text { Letras minúsculas comparam as variáveis que estão nas colunas e as } \\
\text { maiúsculas, as das linhas. Letras iguais apontam não significância e as } \\
\text { diferentes, que houve significância estatística entre os dados obtidos }\end{array}$ \\
\hline
\end{tabular}

\begin{tabular}{|c|c|c|}
\hline \multirow[b]{2}{*}{ Tratamento } & \multicolumn{2}{|c|}{ Cura } \\
\hline & Sim & Não \\
\hline Massagem & 1,000 & 0,000 \\
\hline Sondagem & 0,956 & 0,044 \\
\hline$Z: 1,03(p>0,05)$ & & \\
\hline
\end{tabular}

atenção dos pais ou não se tratar de ONLC verdadeira, mas sim, obstrução da drenagem lacrimal secundária aos processos de hipertrofia da mucosa nasal, muito freqüentes em crianças e denominadas de pseudo-obstrução ${ }^{(12)}$.

A maioria das crianças é tratada inicialmente por pediatras e, em não havendo resolução da epífora, referidas para os oftalmologistas. Provavelmente este fato levou a ter $50,0 \%$ da amostra estudada com idade superior a 6 meses quando da procura do Serviço. Assim, esta amostra é não casual, diferindo dos estudos de Cohort ${ }^{(10)}$, quando os índices de cura se referem a dados populacionais, com amostras casualizadas.

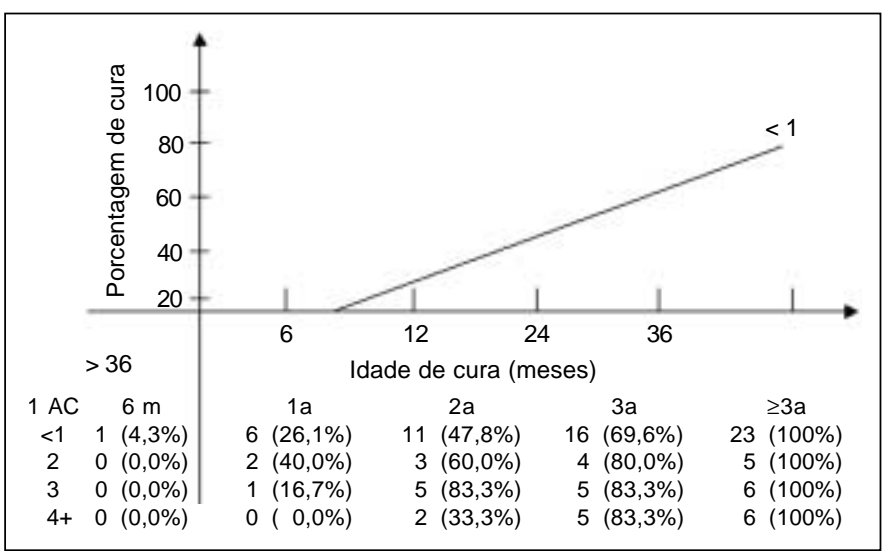

Gráfico 1 - Porcentagem de cura segundo idade do início do lacrimejamento e idade de cura

Avaliando-se a conduta terapêutica, observa-se que a maioria das crianças necessitaram sondagem da via lacrimal. Este fato deve ser reflexo da idade da amostra ao chegar no Serviço, em época que já justifica a sondagem, uma vez que a literatura sobre o assunto é farta de relatos de que a porcentagem de cura espontânea é menor com o aumento da idade da criança $a^{(9,13)}$.

Existem várias controvérsias com relação a melhor idade para sondagem. É nossa conduta usar o tratamento conservador (massagem de Criegler) até aproximadamente 1 ano de vida. Os defensores da sondagem precoce acreditam que postergá-la resultaria na diminuição do índice de sucesso, devido à fibrose e aderências causadas pela inflamação crônica, além de ser mais barato pois pode ser realizada no próprio consultório, sem a necessidade de anestesia geral ${ }^{(6-7)}$.

Porém, na nossa experiência, existem vantagens que justificam a sondagem tardia. A grande maioria das ONLC (até 95\% delas) podem se resolver espontaneamente, até o primeiro ano de vida ${ }^{(13-15)}$ e, mesmo com a idade de 2 anos, $69 \%$ das ONLC ainda podem desaparecer sem sondagem ${ }^{(16)}$, o que também foi possível observar no presente estudo.

Interessante notar que o índice de cura aqui obtido foi elevado, mesmo em crianças maiores - $25 \%$ das crianças que evoluíram para a cura tinham mais de 3 anos de idade. Estudos recentes relatam que crianças menores que 1 ano, de 1 a 2 anos ou maiores que 2 anos, possuem índices semelhantes de insucesso da sondagem e aconselham que se aguarde até que a idade seja maior do que 1 ano para realização deste procedimento ${ }^{(8)}$.

No presente estudo, a cura da epífora foi mais freqüente (50\% de cura) nas crianças menores que 6 meses. Outros observaram 77,6\% de resolução com 3 meses de idade, $91,1 \%$ com 6 meses e 97,4\% com 1 ano de idade ${ }^{(14)}$. Em outro estudo, $50 \%$ dos casos se resolveram aos 4 meses de idade ${ }^{(13)}$.

A avaliação da proporção de cura com a massagem, em relação à idade do início da epífora mostrou que, quando a epífora se inicia antes dos 2 meses de vida, há maior chance de cura com a massagem. Provavelmente, o início mais tardio pode ser decorrente de outras afecções que não a ONLC ${ }^{(12)}$, 
piorando o prognóstico, como evidenciado no presente estudo que mostrou menor chance de cura nas epíforas tardias.

Da mesma forma, a avaliação de cura com a sondagem relacionada à idade do início da epífora mostrou que a possibilidade de cura será menor nas crianças que iniciaram o sintoma mais tardiamente.

$\mathrm{O}$ teste das proporções binomiais mostrou que a chance de cura com a massagem é semelhante ao da sondagem, o que justificaria a preferência da massagem, evitando os riscos inerentes à anestesia geral para a sondagem.

Assim, a sondagem ficaria reservada para os casos refratários ao tratamento conservador, podendo ser realizada até após os 2 anos de vida, com chance de resolução com massagem em $60 \%$ dos casos até os 2 anos de idade e índice de cura de $80 \%$ com sondagem dos 12 aos 14 meses, comparados com $31 \%$ sem tratamento ${ }^{(10)}$.

Porém, a aparente vantagem da sondagem não significa que ela deva ser o tratamento de escolha para todos os pacientes, uma vez que toda intervenção cirúrgica acarreta certo risco, como trauma da via lacrimal que poderia estar destinada à resolução espontânea ${ }^{(10)}$.

Usando meta-análise, estudaram-se todas as publicações a respeito da ONLC, concluindo-se que os casos resistentes à sondagem constituem menos que $2 \%$ de todos os portadores sintomáticos e esta pequena fração não deve influenciar o tratamento dos outros 98\%, uma vez que este insucesso à sondagem possa ser resultado de auto-seleção de casos não resolvidos espontaneamente ${ }^{(15)}$.

Índices de cura com a sondagem de aproximadamente 90\% dos casos são relatados em crianças maiores de 13 meses $^{(17)}$ ou de $73,3 \%$ em maiores de 24 meses $^{(18)}$. A sondagem e a irrigação da via lacrimal ocasionou $96,8 \%$ de cura em crianças acima de 3 anos de idade ${ }^{(19)}$, fazendo com que se reconsidere opiniões sobre piora do prognóstico quando se retarda a sondagem.

Mais importante que a idade da criança na época da sondagem, fatores preditivos de prognóstico ruim seriam: mucoceles grandes, epífora ou secreção abundantes, epífora de início tardio e bilateral, falha no tratamento com massagem ou sondagem, presença de obstáculo rígido ("hard stop") quando se tenta a sondagem ${ }^{(11,17)}$.

\section{CONCLUSÃO}

Os dados obtidos nos permitem concluir que a chance de cura é menor quando a epífora se inicia tardiamente. A cura com a massagem ou com a sondagem ocorre em proporções semelhantes. A resolução da epífora é possível em crianças mais velhas, tendo sido observada até em crianças acima dos 3 anos de idade. Portanto, nossos resultados fortalecem a conduta da sondagem tardia, uma vez que há possibilidade de cura espontânea em crianças maiores, evitando-se, assim, procedimentos desnecessários.

\section{ABSTRACT}

Purpose: To evaluate the possibility of epiphora relief in congenital nasolacrimal obstruction according to the age of the beginning of epiphora complaint and treatment effectiveness. Methods: Forty children with congenital nasolacrimal obstruction underwent treatment between 1997 and 1999 and were evaluated according to: age of start of epiphora, the results of the treatment and the possibility of successful outcome. The data were submitted to statistical analysis by the binomial proportional test between and within populations. Results: The success rate was worst when the epiphora started after the age of 4 months and results were similar on massage and probing, cure occurring even beyond 3 years of age. Conclusion: According to our results, the relief of epiphora was worst when starting at age over 4 months. Massage and probing may have good results even in children older than 3 years.

Keywords: Lacrimal duct obstruction/congenital; Lacrimal duct obstruction/therapy; Nasolacrimal duct; Massage; Outcome results

\section{REFERÊNCIAS}

1. Guerry D III, Kending EJ Jr. Congenital impotency of the nasolacrimal duct. Arch Ophthalmol. 1948;39:193-294.

2. MacEwen CJ, Young JD. Epiphora in the first year of life. Eye. 1991;5(Pt 5): 596-600.

3. Gonçalves Dias AK, Soccol O, Cunha M, Gomes JAP, Pinheiro RK, Peres MFP. Freqüência de obstrução congênita do ducto nasolacrimal na clínica pediátrica da Santa Casa de São Paulo. Arq Bras Oftalmol. 1994;57(2):118-20.

4. Noda S, Hayasaka S, Setogawa T. Congenital nasolacrimal obstruction in Japanese infants: its incidence and treatment with massage. J Pediatr Ophthalmol Strabismus. 1991;28(1):20-2.

5. Nucci P, Capoferri C, Alfarano R, Brancato R. Conservative management of congenital nasolacrimal duct obstruction. J Pediatr Strabismus. 1989;26(1):39-43.

6. Rehurek J, Holousova M. Optimization of therapy in lacrimal duct obstruction in neonates and infants. Cesk Slov Oftalmol. 1997;53(6):391-4.

7. Goldblum TA, Summers CG, Egbert JE, Letson RD. Office probing for congenital nasolacrimal duct obstruction: a study of parental satisfaction. J Pediatr Ophthalmol Strabismus. 1996;33(4):244-7.

8. Zwaan J. Treatment of congenital nasolacrimal duct obstruction before and after the age of 1 year. Ophthalmic Surg Lasers. 1997;28(11):932-6.

9. Petrelli RL. Pediatric nasolacrimal obstruction: update on management. Conn Med. 1985;49(12):782-4.

10. Young JD, MacEwen CJ, Ogston SA. Congenital nasolacrimal duct obstruction in the second year of life: a multicentre trial of management. Eye. 1996; 10(Pt 4):485-91.

11. Schellini SA, Gaiotto PC, Schellini RC, Silva MRB. Obstrução nasolacrimal congênita- diagnóstico e tratamento. Rev Bras Oftalmol. 1994;53(5):47-53.

12. Schellini SA, Silva MRB, Schellini RC. Pseudo-obstrução nasolacrimal na infância. Arq Bras Oftalmol. 1994;57(5):348-51.

13. Petersen RA, Robb RM. The natural course of congenital obstruction of the nasolacrimal duct. J Pediatr Ophthalmol Strabismus. 1978;15(4):246-50.

14. Price HW. Dacrioestenosis. J Pediatr. 1947;30:302-5.

15. Paul TO, Shepherd R. Congenital nasolacrimal duct obstruction: natural history and the timing of optimal intervention. J Pediatr Ophthalmol Strabismus. 1994;31(6):362-7.

16. Paul OT. Medical management of congenital nasolacrimal duct obstruction. J Pediatr Ophthalmol Strabismus. 1985;22(1):68-70.

17. Yap EY, Yip CC. Outcome of late probing for congenital nasolacrimal obstruction in Singapore children. Int Ophthalmol. 1997-98;21(6):331-4.

18. Honavar SG, Prakash VE, Rao GN. Outcome of probing for congenital nasolacrimal duct obstruction in older children. Am J Ophthalmol. 2000;130(1):42-8.

19. Robb RM. Success rates of nasolacrimal duct probing at time intervals after 1 year of age. Ophthalmology. 1998;105(7):1307-10. 